\title{
Nucleon transfer contribution to the absorptive potential in heavy-ion scattering
}

\author{
Fl. Stancu \\ Institute of Physics, University of Liège, Sart Tilman, B-4000 Liege 1, Belgium \\ D. M. Brink \\ Theoretical Physics Department, University of Oxford, Oxford, United Kingdom
}

(Received 6 August 1981)

\begin{abstract}
We propose a simple model for estimating the nucleon transfer contribution to the imaginary part of the nucleus-nucleus optical potential. Using the proximity method we calculate the transfer probability per unit time in terms of the flux of nucleons between two slabs. Pauli blocking and barrier penetration effects are taken into account. We compare our results with phenomenological potentials at the strong absorption radius.
\end{abstract}

$\left[\begin{array}{c}\text { NUCLEAR REACTIONS Imaginary part of the heavy ion optical po- } \\ \text { tential. Nucleon transfer contribution. Barrier penetration, Pauli block- } \\ \text { ing, and relative motion effects. }\end{array}\right]$

\section{INTRODUCTION}

The imaginary part $W$ of the optical potential describes the depopulation of the entrance channel in elastic heavy ion scattering. In a recent article Broglia et al. ${ }^{1}$ argue that nucleon transfer between the two nuclei and inelastic excitation of the two nuclei give the most important contributions to $W$ at large separations. They view the total depopulation of the entrance channel as due to elementary transitions so that in their picture multinuclear transfer is the result of the successive transfer of individual nucleons. If it is assumed that these elementary transitions are independent of each other and that the probability of any specific transition occurring during the collision is small, then the total absorptive potential $W$ can be written as an incoherent sum of contributions from the different elementary processes. Broglia et al. ${ }^{1}$ write $W=W_{\text {inel }}+W_{\text {trans }}$, where $W_{\text {inel }}$ is the contribution of inelastic excitation to $W$, and $W_{\text {trans }}$ is the nucleon transfer contribution. They claim that the range of $W_{\text {trans }}$ is larger than that of $W_{\text {inel }}$. Accepting this point of view $W_{\text {trans }}$ would give a major contribution to $W$ around the strong absorption radius.

In the present work we propose a simple method for calculating $W_{\text {trans }}$. The nuclei are treated by the Fermi gas model and the flux of nucleons from one nucleus to the other is calculated by taking into account the Pauli allowed region in the momentum space and the tunneling through the barrier formed between the single particle wells.

In Sec. II we define $W_{\text {trans }}$ in terms of the flux. In Sec. III we calculate the flux from our model. In Sec. IV the present results are compared to the phenomenological imaginary potentials of various pairs at the strong absorption radius. The last section is devoted to the conclusions.

\section{THE TRANSFER CONTRIBUTION TO $W$}

We write the optical potential for scattering of two nuclei $A$ and $B$ as $V(\overrightarrow{\mathrm{r}})-i W(\overrightarrow{\mathrm{r}})$ where $\overrightarrow{\mathrm{r}}$ is the distance between their centers. Throughout this work we assume that the nuclei are spherical. If the scattering wave function is $\psi(\vec{r})$ then the probability density for finding the nuclei $A$ and $B$ in their ground states with relative coordinate $\vec{r}$ is $\rho(\vec{r})=\psi^{*}(\vec{r}) \psi(\vec{r})$ and from the time dependent Schrödinger equation it follows that

$$
\frac{d}{d t} \int \rho(\overrightarrow{\mathrm{r}}) d^{3} r=-\frac{2}{\hbar} \int W(\overrightarrow{\mathrm{r}}) \rho(\overrightarrow{\mathrm{r}}) d^{3} r .
$$

Thus $2 W(r) / \hbar$ is the transition probability per unit time for a transition from the elastic channel when the relative coordinate of the two nuclei is $|\overrightarrow{\mathbf{r}}|$.

In order to calculate $W_{\text {trans }}$ we need the probability per unit time for transfer of a nucleon between the two nuclei. This can be found by the proximity 
method $^{2}$ which was used by Randrup for calculating the friction coefficient ${ }^{3}$ and mass transport coefficients ${ }^{4}$ for deep inelastic collisions. Instead of two finite nuclei one considers two slabs of semi-infinite nuclear matter with surfaces separated by a distance $s$ [cf. Fig. 1(a)]. Let $\phi_{B A}(s)$ be the flux of nucleons from the slab $A$ to the slab $B$, and $\phi_{A B}(s)$ be the corresponding flux from $B$ to $A$.

Now consider two finite nuclei with radii $R_{A}$ and $R_{B}$ with a minimum separation $s$ between their surfaces [Fig. 1(b)]. The proximity method ${ }^{2}$ gives the transition probability per unit time for transfer of a nucleon between the two nuclei as $2 \pi \bar{R} I(s)$, where

$$
\bar{R}=R_{A} R_{B} /\left(R_{A}+R_{B}\right)
$$

with $R_{A(B)}$ to be defined later and

$$
I(s)=\int_{s}^{\infty} d s^{\prime}\left[\phi_{B A}\left(s^{\prime}\right)+\phi_{A B}\left(s^{\prime}\right)\right] .
$$

The quantity $2 \pi \bar{R} I(s)$ is the integrated flux and gives the probability per unit time for depopulation of the ground state by nucleon transfer. According to Eq. (1) this is the same quantity as $2 W_{\text {trans }} / \hbar$. Hence we obtain the relation

$$
W_{\text {trans }}=\pi \hbar \bar{R} I(s) .
$$

Let $V^{q}(z, s)$ denote the one-dimensional barrier through which a nucleon $(q=n$ for a neutron and $q=p$ for a proton) has to tunnel when it passes between the slabs $A$ and $B$. In a semiclassical approximation ${ }^{5}$ the transmission coefficient for a nucleon with momentum component $\hbar k_{z}$ in the slab $A$ is given by

$$
P^{q}\left(k_{z}, s\right)=1 /\left[1+\exp \left(2 Y^{q}\right)\right],
$$

where the penetrability integral is

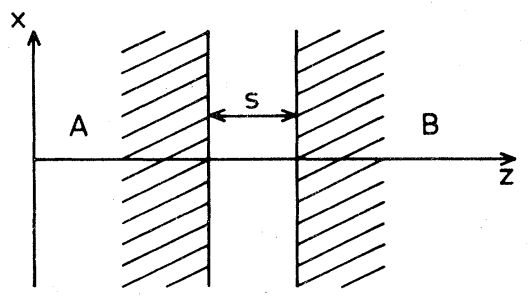

(a)

$$
Y^{q}=\int_{z_{1}}^{z_{2}}\left[\frac{2 m}{\hbar^{2}} V(z)-k_{z}{ }^{2}\right]^{1 / 2} d z .
$$

Equation (5) reduces to the standard WentzelKramers-Brillouin (WKB) formula when $Y^{q}$ is large and is a better approximation for energies near the top of the barrier.

The formula for the flux of nucleons of type $q$ from $A$ into $B$ is given by

$$
\begin{aligned}
\phi_{B A}^{q}(s)=\frac{2}{(2 \pi)^{3}} \int_{k_{z}>0} & d^{3} \overrightarrow{\mathrm{k}} P^{q}\left(k_{z}, s\right) \frac{\hbar k_{z}}{m} \\
& \times n_{A}^{q}(\overrightarrow{\mathrm{k}})\left[1-n_{B}^{q}(\overrightarrow{\mathrm{k}})\right],
\end{aligned}
$$

where $n_{A}^{q}(\overrightarrow{\mathrm{k}})\left[n_{B}^{q}(\overrightarrow{\mathrm{k}})\right]$ is the occupation probability of the state $\overrightarrow{\mathrm{k}}$ in $A(B)$. The factor $\left[1-n_{B}^{q}(\overrightarrow{\mathrm{k}})\right]$ takes into account the effects of the Pauli principle in the final state. The flux $\phi_{A B}^{q}(s)$ from $B$ into $A$ is given by a formula similar to Eq. (7) with $A$ and $B$ interchanged and $k_{z}$ replaced by $-k_{z}$.

\section{PROXIMITY FORM FOR THE TRANSITION PROBABILITY}

In a general case the neutron and proton potentials $V^{q}(z, s)$ can be different and, therefore, neutrons and protons can give different contributions to $W_{\text {trans }}$. In this section we restrict the discussion to a special case where we take equal number of neutrons $N$ and protons $Z$, i.e.,

$$
N_{A}=Z_{A}=N_{B}=Z_{B},
$$

and where Coulomb effects are neglected. Then the neutrons and protons have identical single particle levels. The potential barrier $V^{q}(z, s)$ is symmetric and the fluxes are all equal

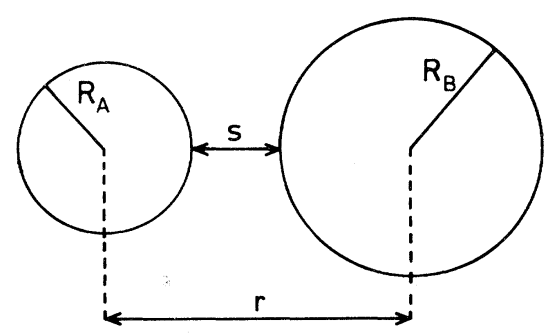

(b)

FIG. 1. Illustration of the proximity method. (a) Two semi-infinite slabs of nuclear matter separated by a distance $s$ along the $z$ axis. (b) Two finite nuclei of radii $R_{A}$ and $R_{B}$ separated by the distance $r$ between their centers. The shortest distance between their surfaces is $s=r-R_{A}-R_{B}$. 
$\phi_{A B}^{n}(s)=\phi_{B A}^{n}(s)=\phi_{A B}^{p}(s)=\phi_{B A}^{p}(s)=\frac{1}{2} \phi(s)$.

In this case $W_{\text {trans }}$ is given by Eq. (4) with

$$
I(s)=2 \int_{s}^{\infty} \phi\left(s^{\prime}\right) d s^{\prime} .
$$

For simplicity, from now on we shall drop the upper index $q$.

We take a potential of the form

$$
\begin{aligned}
V(z, s)= & V_{0}-\frac{V_{0}^{A}}{1+\exp \left[\left(\frac{1}{2} s-z\right) / a\right]} \\
& -\frac{V_{0}^{B}}{1+\exp \left[\left(\frac{1}{2} s+z\right) / a\right]},
\end{aligned}
$$

with $V_{0}^{A}=V_{0}^{B}=V_{0}=50 \mathrm{MeV}$ and $a=0.65 \mathrm{fm}$. For the Fermi wave number we choose $k_{F}=1.36 \mathrm{fm}^{-1}$. These parameters are consistent with the systematics given by Bohr and Mottelson ${ }^{6}$ for the nucleonnucleus scattering data. They have been used recently by Brosa and Gross ${ }^{7}$ for calculating the mass flow in heavy ion deep inelastic scattering. With these restrictions the formula for $\phi(s)$ reduces to

$$
\begin{array}{rl}
\phi(s)=\frac{4}{(2 \pi)^{3}} \frac{\pi \hbar}{m} \int_{0}^{k_{F}} & d k_{z} P\left(k_{z}, s\right) \\
& \times\left(k_{F}{ }^{2}-k_{z}{ }^{2}\right) k_{z} g\left(k_{z}, v\right) .
\end{array}
$$

The factor $g\left(k_{z}, v\right)$ in Eq. (11) contains the effects of the Pauli exclusion principle. We assume that the slab $B$ moves relative to $A$ in the $x$ direction with speed $v$. Then

$$
\begin{aligned}
n_{A}(\overrightarrow{\mathrm{k}}) & =1, \text { if }\left|\overrightarrow{\mathrm{k}}+\frac{\overrightarrow{\mathrm{q}}}{2}\right|<k_{F} \\
& =0, \text { if }\left|\overrightarrow{\mathrm{k}}+\frac{\overrightarrow{\mathrm{q}}}{2}\right|>k_{F},
\end{aligned}
$$

while

$$
\begin{aligned}
n_{B}(\overrightarrow{\mathrm{k}}) & =1, \text { if }\left|\overrightarrow{\mathrm{k}}-\frac{\overrightarrow{\mathrm{q}}}{2}\right|<k_{F} \\
& =0, \text { if }\left|\overrightarrow{\mathrm{k}}-\frac{\overrightarrow{\mathrm{q}}}{2}\right|>k_{F},
\end{aligned}
$$

where $\overrightarrow{\mathrm{q}}=m v \overrightarrow{\mathrm{i}} / \hbar$. Figure 2 shows a cross section of the functions $n_{A}(\overrightarrow{\mathrm{k}})$ and $n_{B}(\overrightarrow{\mathrm{k}})$ at a fixed value of $k_{z}$. The shaded region indicates states which are occupied in $A$ and not occupied in $B$ as in Eq. (7). They give the values of $k_{x}$ and $k_{y}$ for which transitions from $A$ to $B$ are allowed by the Pauli principle. The factor $g$ lies in the range $0 \leq g \leq 1$ and is proportional to the shaded area in Fig. 2. In other

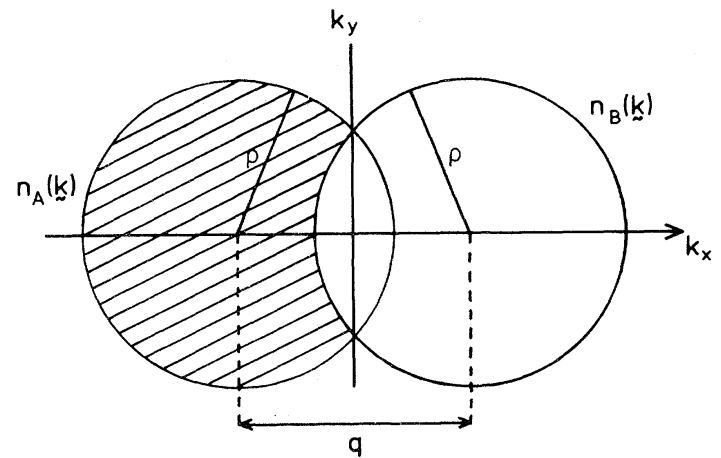

FIG. 2. The Pauli allowed region for nucleon transfer at given $k_{z}$. The shaded region is allowed for transitions from $A$ to $B$. The area allowed for transitions from $B$ to $A$ belongs to the other circle. Both circles are of equal radii $\rho=\left(k_{F}{ }^{2}-k_{z}{ }^{2}\right)^{1 / 2}$ and their centers are separated by the relative momentum per nucleon $q$.

words $g$ is given by the shaded area divided by $\pi \rho^{2}$ where $\rho=\left(k_{F}{ }^{2}-k_{z}{ }^{2}\right)^{1 / 2}$ is the radius of the circle shown in the figure. Therefore we have:

$g\left(k_{z}, v\right)= \begin{cases}1, & \text { if } q>2 \rho \\ \frac{2}{\pi}\left(\alpha+\frac{1}{2} \sin 2 \alpha\right) ; & \alpha=\sin ^{-1}(q / 2 \rho) . \\ & \text { if } q<2 \rho\end{cases}$

Except at the point of closest approach the relative velocity of the interacting nuclei has two components, one radial-along the direction connecting the linear centers in coordinate space, and the other tangential-perpendicular to the first. The formulas (11) and (12) contain only the contribution of the tangential component. Our calculations should be valid when the tangential component is large compared with the radial component at separations where the transfer is important. Thus our result can be used when the incident energy is well above the Coulomb barrier. It does not apply for energies near or below the Coulomb barrier when the radial motion is more important than the tangential motion. A practical criterion for "well above the Coulomb barrier" would be a grazing angle $\theta_{g} \lesssim 90^{\circ}$. This condition is well fulfilled in all cases given in Table I except for ${ }^{16} \mathrm{O}+{ }^{58} \mathrm{Ni}$ at $E_{\text {lab }}=45 \mathrm{MeV}$.

Figure 3 shows values of the flux $\phi(s)$ for several different values of $q$ calculated with the parameters given after Eq. (10). The flux is zero if the relative velocity is zero because the Pauli blocking effect is complete. When $v$ is small $\phi$ is approximately proportional to the relative velocity. At $q=2 k_{F}$ the 
TABLE I. Calculated $W_{\text {trans }}$ and phenomenological $W_{\exp }$ potentials for various systems at the strong absorption radius $D_{1 / 2}$ and $E_{\text {lab }}$ (or $E_{\text {c.m. }}$ ). The other notations are $q$, the relative momentum per nucleon; $s=D_{1 / 2}-R_{A}-R_{B}$, defined as in Eqs. (16) and (17); $\bar{R}$ is given by Eqs. (2) and (16); $W_{0}$ the depth, $R_{W}$ the radius, and $a_{W}$ the thickness parameters of the phenomenological imaginary potentials taken from the references indicated in the last column.

\begin{tabular}{|c|c|c|c|c|c|c|c|c|c|c|c|c|}
\hline System & $\begin{array}{c}E_{\text {lab }} \\
(\mathrm{MeV})\end{array}$ & $\begin{array}{l}E_{\text {c.m. }} \\
(\mathrm{MeV})\end{array}$ & $\begin{array}{c}q / 2 \\
\left(\mathrm{fm}^{-1}\right)\end{array}$ & $\begin{array}{c}s \\
(\mathrm{fm})\end{array}$ & $\begin{array}{c}\bar{R} \\
(\mathrm{fm})\end{array}$ & $\begin{array}{l}W_{\text {trans }} \\
(\mathrm{MeV})\end{array}$ & $\begin{array}{c}W_{\exp }\left(D_{1 / 2}\right) \\
(\mathrm{MeV})\end{array}$ & $\begin{array}{l}D_{1 / 2} \\
(\mathrm{fm})\end{array}$ & $\begin{array}{c}W_{0} \\
(\mathrm{MeV})\end{array}$ & $\begin{array}{c}R_{W} \\
(\mathrm{fm})\end{array}$ & $\begin{array}{c}a_{W} \\
(\mathrm{fm})\end{array}$ & Ref. \\
\hline \multirow[t]{3}{*}{${ }^{16} \mathrm{O}+{ }^{40} \mathrm{Ca}$} & 55.6 & 39.7 & 0.13 & 2.3 & 1.81 & 0.12 & 0.32 & 9.7 & 12.9 & 7.72 & 0.54 & 9 \\
\hline & 103.6 & 74.0 & 0.23 & 1.9 & & 0.40 & 0.67 & 9.3 & 13.2 & & & \\
\hline & 214.1 & 152.9 & 0.37 & 1.6 & & 0.90 & 1.18 & 9.0 & 13.8 & & & \\
\hline \multirow[t]{3}{*}{${ }^{16} \mathrm{O}+{ }^{58} \mathrm{Ni}$} & 45 & 35.3 & 0.05 & 2.0 & 1.91 & 0.08 & $0.12_{-0.04}^{+0.20}$ & 10.0 & & & & 10 \\
\hline & 60 & 47.0 & 0.12 & 1.9 & & 0.24 & $0.26_{-0.14}^{+0.21}$ & 9.9 & & & & \\
\hline & 81 & 63.5 & 0.17 & 1.6 & & 0.57 & $0.62 \pm 0.18$ & 9.6 & & & & \\
\hline${ }^{16} \mathrm{O}+{ }^{60} \mathrm{Ni}$ & 142 & 112.1 & 0.27 & 1.6 & 1.92 & 0.86 & $1.13 \pm 0.17$ & 9.6 & & & & 10 \\
\hline \multirow[t]{3}{*}{${ }^{16} \mathrm{O}+{ }^{208} \mathrm{~Pb}$} & 129.5 & 120.3 & 0.19 & 2.2 & 2.21 & 0.26 & 0.89 & 12.8 & 19.4 & 10.98 & 0.60 & 9 \\
\hline & 192 & 178.3 & 0.29 & 1.8 & & 0.69 & 1.34 & 12.4 & 15.6 & & & \\
\hline & 312.6 & 290.3 & 0.41 & 1.5 & & 1.23 & 1.53 & 12.1 & 11.4 & & & \\
\hline${ }^{20} \mathrm{Ne}+{ }^{40} \mathrm{Ca}$ & 151 & 100.7 & 0.25 & 2 & 1.89 & 0.40 & 0.94 & 9.7 & 40 & 7.13 & 0.68 & 11 \\
\hline${ }^{32} S+{ }^{32} S$ & 90.9 & 45.4 & 0.08 & 2.2 & 1.98 & 0.11 & 0.37 & 10.1 & 22.7 & 8.25 & 0.45 & 9 \\
\hline \multirow[t]{3}{*}{${ }^{40} \mathrm{Ca}+{ }^{40} \mathrm{Ca}$} & 143.6 & 71.8 & 0.10 & 2.2 & 2.14 & 0.13 & 0.34 & 10.75 & 12.13 & 9.23 & 0.43 & 12 \\
\hline & 186 & 93 & 0.15 & 2 & & 0.20 & 0.37 & 10.72 & & & & \\
\hline & 240 & 120 & 0.20 & 1.9 & & 0.34 & 0.48 & 10.6 & & & & \\
\hline \multirow[t]{2}{*}{${ }^{136} \mathrm{Xe}+{ }^{209} \mathrm{Bi}$} & 940 & 569.4 & 0.14 & 1.6 & 3.44 & 0.73 & 2.03 & 15.5 & 15.94 & 14.71 & 0.41 & 13 \\
\hline & 1130 & 684.6 & 0.19 & 1.3 & & 1.54 & 0.97 & 15.2 & 6.95 & 14.4 & 0.44 & \\
\hline
\end{tabular}

Pauli blocking has no effect anymore and the flux reaches a maximum. The latter case has also been considered by Ko, Bertsch, and Cha. ${ }^{8}$ They calculate the flux with slightly different parameters than ours and use it for estimating the diffusion coefficient of a transport equation.

In Fig. 4 we present results for $\frac{1}{2} I(s)$ defined by Eq. (9) as a function of $s$ for a selection of values of the relative momentum per nucleon $q$. Both $\phi(s)$ and $\frac{1}{2} I(s)$ can be parametrized by simple analytic formulas in terms of $s$ and $q$. From Figs. 3 and 4 we notice that for $s$ greater than $s_{0}=2.5 \mathrm{fm}, \phi$ and $\frac{1}{2} I$ have an exponential decay. Introducing the variable

$$
\beta=\frac{q}{2 k_{F}}=\frac{m v}{2 \hbar k_{F}}
$$

we found that $\frac{1}{2} I(s)$ can be most conveniently parametrized by

$$
10^{3} \times \frac{1}{2} I(s)=\left\{\begin{array}{l}
\beta^{2} I_{0}(s)+\beta(1-\beta) I_{1}(s) s<s_{0}=2.5 \mathrm{fm}, \\
{\left[0.129 \beta^{2}+0.406 \beta(1-\beta)\right] e^{-1.8\left(s-s_{0}\right)} s \geq s_{0},}
\end{array}\right.
$$

where

$$
\begin{aligned}
I_{0}= & 0.129-0.232\left(s-s_{0}\right)+0.209\left(s-s_{0}\right)^{2} \\
& -0.091\left(s-s_{0}\right)^{3}+0.091\left(s-s_{0}\right)^{4}, \\
I_{1}= & 0.406-0.731\left(s-s_{0}\right)+0.657\left(s-s_{0}\right)^{2} \\
& -0.262\left(s-s_{0}\right)^{3}+0.106\left(s-s_{0}\right)^{4} .
\end{aligned}
$$

The parametrization for $\phi$ can be obtained by taking the derivative of $\frac{1}{2} I(s)$. The polynomial $I_{0}$ has been fitted to reproduce the numerical results for $q=2 k_{F}$ and $I_{1}$ fits well results at small $q$.

The analytical expressions (14) and (15) can be easily used for any pair of nuclei in the way indicated by Randrup. ${ }^{3,4}$ The only difference consists in the definition we adopt for $\boldsymbol{R}_{A(B)}$. Here $\boldsymbol{R}_{A(B)}$ 


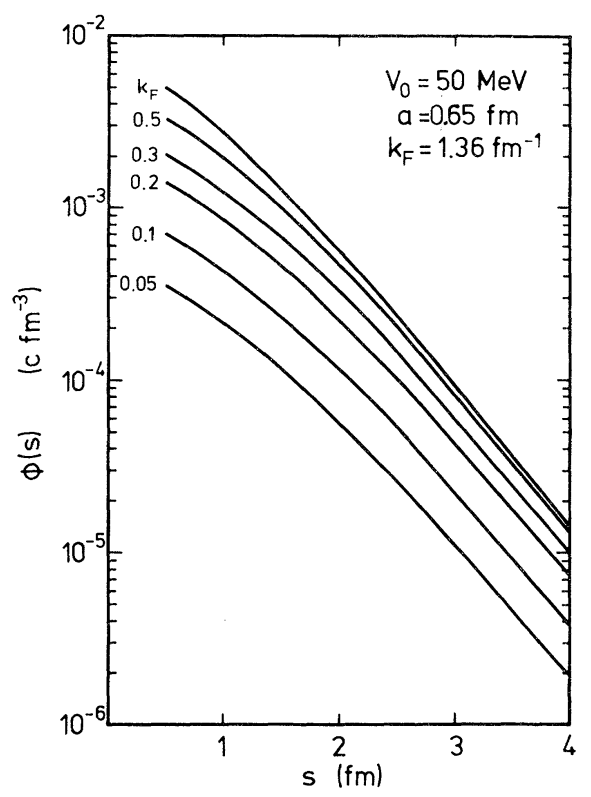

FIG. 3. The flux $\phi$ of nucleons from one slab to the other as a function of the spearation distance $s$ for several values of $q / 2$. The parameters of the barrier (10) are $V_{0}=50 \mathrm{MeV}$ and $a=0.65 \mathrm{fm}$. The Fermi momentum is $k_{F}=1.36 \mathrm{fm}^{-1}$.

represents the nucleon-nucleus potential radius and not the central radius as in the proximity method. ${ }^{2}$ To be consistent with the other parameters of the model we choose this according to Bohr and Mottelson $^{6}$

$$
\begin{aligned}
& R_{i}=r_{0} A_{i}{ }^{1 / 3} ; \\
& r_{0}=1.25 \mathrm{fm}(i=A, B) .
\end{aligned}
$$

In the following section we shall use $\frac{1}{2} I(s)$ to the calculation of $W_{\text {trans }}(r)$ where $s$ and $r$ are related by

$$
s=r-R_{A}-R_{B} \text {. }
$$

\section{COMPARISON TO PHENOMENOLOGICAL POTENTIALS}

Although the present version of our model is restricted to identical nuclei we shall apply it to a larger variety of pairs and then discuss its limitations.

Taking into account the units of $I(s)$ the formula (4) becomes

$$
W_{\text {trans }}(r)=620 \bar{R} I(s)(\mathrm{MeV}),
$$

where $\bar{R}$ and $s$ have been defined in Eqs. (2) and

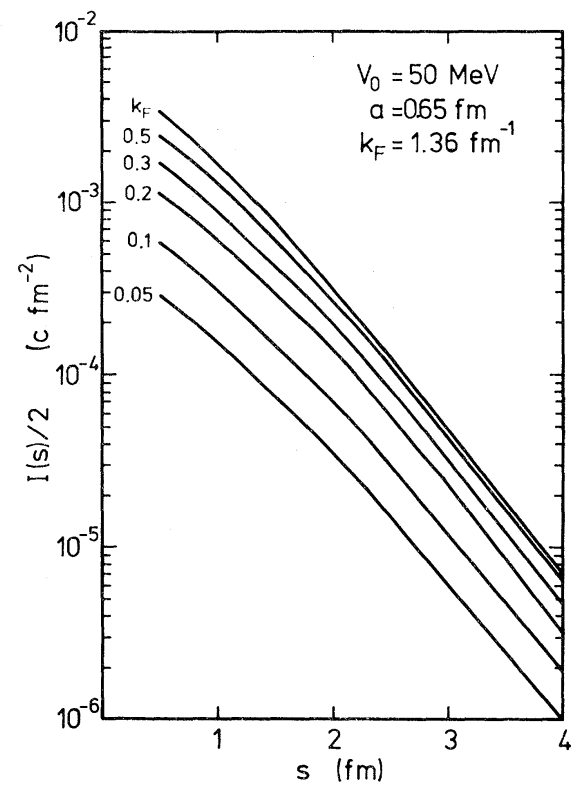

FIG. 4. The zeroth incomplete moment $\frac{1}{2} I$ of the flux $\phi$ defined by Eq. (9) as a function of $s$ for a series of values of $q / 2$. The parameters are the same as in Fig. 4.

(17), respectively, and the numerical value of $\frac{1}{2} I(s)$ can be read from Fig. 4 or calculated from the expressions (14) and (15).

According to Ref. 9 the elastic scattering cross section having a Fresnel pattern only determines the optical potential in the vicinity of the strong absorption radius. We therefore compare our calculated values of $W_{\text {trans }}$ with the imaginary part of the phenomenological potential at the strong absorption radius $D_{1 / 2}$. This comparison is given in Table I. Two general remarks can be made. Our model gives the right order of magnitude and an exponential decrease with a constant $\gamma=1.8 \mathrm{fm}^{-1}$ consistent with the surface thickness $a$ of the imaginary phenomenological potential, i.e., $a \sim 1 / \gamma=0.55 \mathrm{fm}$.

In the peripheral region one expects that two types of phenomena contribute to the absorption from the incident channel. These are the transfer of nucleons and the inelastic transitions. ${ }^{1}$ According to this point of view $W_{\text {trans }}$ would represent only a part of the phenomenological imaginary potential. One would therefore expect $W_{\text {trans }}$ to be similar or smaller than the phenomenological potential. With one exception this is the case for all examples we give in Table I. The exception is made by ${ }^{136} \mathrm{Xe}+$ ${ }^{209} \mathrm{Bi}$ at $E_{\mathrm{lab}}=1130 \mathrm{MeV}$ and we believe that the disagreement is due to the inclusion of some inelas- 
tic events in the measured elastic cross section.

Our calculations give an energy dependent imaginary potential. This is a consequence of the effect of the Pauli principle expressed in Eqs. (11) and (12). The examples shown in Table I indicate a similarity between the calculated energy dependence and the trend shown by the experimental data. For ${ }^{16} \mathrm{O}+{ }^{40} \mathrm{Ca},{ }^{16} \mathrm{O}+{ }^{58} \mathrm{Ni}$, and ${ }^{40} \mathrm{Ca}+{ }^{40} \mathrm{Ca}$ at the highest energy given in the table, $W_{\text {trans }}$ and $W_{\text {exp }}$ are very similar. For the lowest energy the calculated potential is generally about half of the experimental value.

In making the comparison one should bear in mind that there are uncertainties both in the extraction of $W_{\text {exp }}$ and the calculation of $W_{\text {trans }}$. The case of ${ }^{16} \mathrm{O}+\mathrm{Ni}$ shows the order of magnitude of the uncertainties expected in $W_{\text {exp }}$. The calculated values of $W_{\text {trans }}$ depend upon the choice of the parameters $V_{0}, a, k_{F}$, and $r_{0}$. For example, reducing $V_{0}$ to $45 \mathrm{MeV}$ increases $W_{\text {trans }}$ by about a factor of 2 at $s=2 \mathrm{fm}$. Reducing $a$ by $0.05 \mathrm{fm}$ decreases $W_{\text {trans }}$ by about $25 \%$ for the same value of $s$. The choice of $r_{0}$ affects the value of $s$ at the strong absorption radius [see Eq. (17)] and a small change in $s$ can produce a large modification of $W_{\text {trans }}$.

The pairs ${ }^{40} \mathrm{Ca}+{ }^{40} \mathrm{Ca}$ and ${ }^{32} \mathrm{~S}+{ }^{32} \mathrm{~S}$ correspond to the particular conditions of our model mentioned at the beginning of Sec. III, because these are identical nuclei with $N=Z$. Figure 5(a) shows a typical barrier for such a case. It is calculated for $s=2 \mathrm{fm}$ which is close to the strong absorption radius for several of the examples in Table I. Note that the Fermi level is about $6 \mathrm{MeV}$ above the top of the barrier.

In the following we shall comment on effects which modify the present results. Figures $5(\mathrm{~b})$ and (c) indicate the way in which the barrier is modified if $N_{1}=N_{2}>Z_{1}=Z_{2}$. Here $b$ corresponds to the neutron barrier for ${ }^{208} \mathrm{~Pb}+{ }^{208} \mathrm{~Pb}$. The barrier is still symmetric but has a reduced height because of the isospin dependence of $V_{0}$ for which we choose according to Ref. 7

$$
\begin{aligned}
& V_{0}=-50 \pm 22.5 \frac{N-Z}{A} \\
& \text { (+for neutrons, - for protons). }
\end{aligned}
$$

The neutron Fermi level is raised because $k_{F, n} \sim \rho_{n}{ }^{1 / 3}$. Thus the contribution of neutrons to $W_{\text {trans }}$ is larger than calculated by our simplified model. Figure 5(c) shows the proton barrier for ${ }^{208} \mathrm{~Pb}+{ }^{208} \mathrm{~Pb}$. The height of the barrier is increased because of the isospin dependence of $V_{0}$ and the proton Fermi level is lower and is below the top of

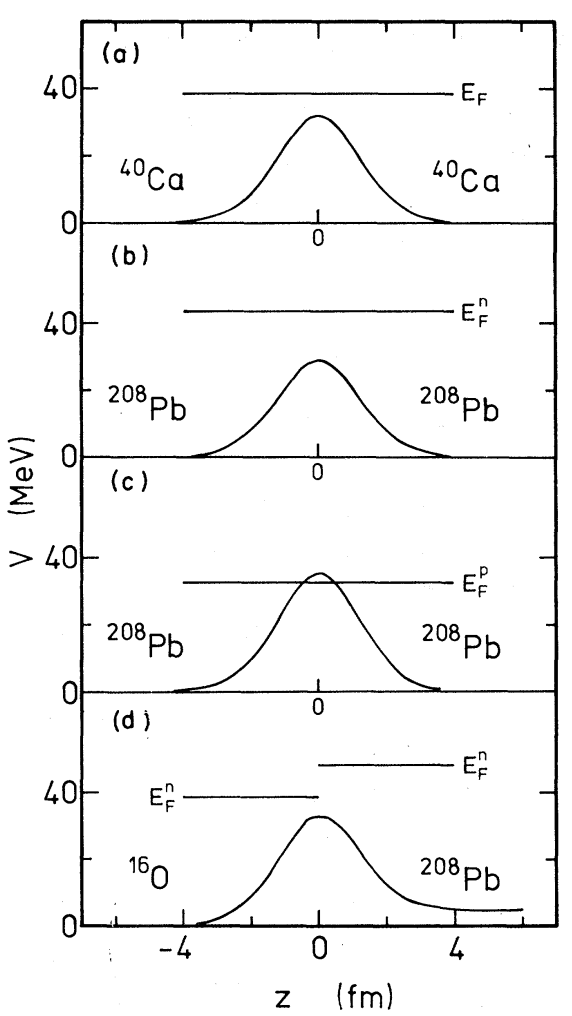

FIG. 5. Nuclear barriers at $s=2 \mathrm{fm}$ and Fermi levels for several pairs of nuclei. The Fermi momentum for nuclear matter is $k_{F}=1.36 \mathrm{fm}^{-1}$. Neutron $(q=n)$ and proton $(q=p)$ Fermi momenta are $k_{F, n}=(2 N / A)^{1 / 3} k_{F}$ and $k_{F, p}=(2 Z / A)^{1 / 3} k_{F}$, respectively. The isospin dependence of the parameter $V_{0}$ of the barrier (10) is given by Eq. (19). The diffuseness parameter is $a=0.65$ fm. (a) Neutron or proton barrier for ${ }^{40} \mathrm{Ca}+{ }^{40} \mathrm{Ca}$ or any other pair of identical nuclei with $N=Z$. (b) Neutron barrier for ${ }^{208} \mathrm{~Pb}+{ }^{208} \mathrm{~Pb}$. (c) Proton barrier for ${ }^{208} \mathrm{~Pb}+{ }^{208} \mathrm{~Pb}$ (no Coulomb interaction). (d) Neutron barrier for ${ }^{16} \mathrm{O}+{ }^{208} \mathrm{~Pb}$; in Eq. (10) we take $V_{0}=V_{0}^{A}$ where $A$ stands for ${ }^{16} \mathrm{O}$.

the barrier. This makes a big reduction in the transfer probability of protons. Such an effect has been considered by Brosa and Gross ${ }^{7}$ in their study of neutron-rich flow contribution to the friction coefficient. They also discuss the hindering role of the Coulomb potential to proton transfer.

Figure 5(d) shows the neutron barrier of ${ }^{16} \mathrm{O}+{ }^{208} \mathrm{~Pb}$. The barrier is asymmetric because of the isospin dependence of $V_{0}$. Also the Fermi levels are different. This implies an asymmetric flux. Moreover, the flux from ${ }^{208} \mathrm{~Pb}$ to ${ }^{16} \mathrm{O}$ is nonzero even for zero relative velocities because there are levels in ${ }^{16} \mathrm{O}$ which can be occupied by transfer. The barrier for proton transfers would also be 
asymmetric and would be modified by the effects of the Coulomb interaction of the protons. ${ }^{7}$

For each specific pair of nuclei it would be interesting to investigate in a quantitative way the modifications resulting from the above mentioned effects.

\section{CONCLUSIONS}

Broglia et al. ${ }^{1}$ have argued that for large separations nucleon transfer gives a major contribution to the imaginary part of the heavy ion optical potential. In the present paper we have made a calculation of $W_{\text {trans }}$ in a simple model. Nucleon transfer between slabs of nuclear matter was studied first and then a connection was made to finite nuclei by using the proximity method. We find that the cal- culated values of $W_{\text {trans }}$ are similar to empirical values of $W$ at the strong absorption radius. There is a dependence of $W_{\text {trans }}$ on energy which resembles the one found experimentally. The dependence of $W_{\text {trans }}$ on the relative distance between the nuclei is exponential at large separation with a surface diffuseness which lies in the range $a=0.45-0.65 \mathrm{fm}$ depending on the parameters used in the calculation. Although the proximity method is a very crude approximation the results look promising. It would be interesting to make a more detailed analysis of the effects neglected in this work.

\section{ACKNOWLEDGMENTS}

This work has been supported by the NATO research Grant No. 1782. One of us (F.S.) would like to thank Dr. P. E. Hodgson for kindly offering hospitality at the Theoretical Nuclear Group of the Oxford University Nuclear Physics Laboratory.
${ }^{1}$ R. A. Broglia, G. Pollarolo, and A. Winther, Nucl. Phys. A361, 307 (1981).

2J. BYocki, J. Randrup, W. J. Swiatecki, and C. F. Tsang, Ann. Phys. (N.Y.) 105, 427 (1977).

3J. Randrup, Ann. Phys. (N.Y.) 112, 356 (1978).

4J. Randrup, Nucl. Phys. A307, 319 (1978).

${ }^{5}$ E. C. Kemble, Phys. Rev. 48, 549 (1935).

${ }^{6}$ A. Bohr and B. R. Mottelson, Nuclear Structure (Benjamin, New York, 1969), Vol. 1, p. 236.

${ }^{7}$ U. Brosa and D. H. E. Gross, Z. Phys. A 298,91 (1980).
${ }^{8}$ C. M. Ko, G. F. Bertsch, and D. Cha, Phys. Lett. 77B, 174 (1978).

${ }^{9}$ G. R. Satchler and W. G. Love, Phys. Rep. 모, 183 (1979).

${ }^{10}$ G. R. Satchler, Phys. Lett. $\underline{58 B}, 409$ (1975).

${ }^{11}$ Nguyen Van Sen, J. C. Gondrand, F. Merchez, and R. Darves-Blanc, Phys. Rev. C 22, 2424 (1980).

${ }^{12} \mathrm{H}$. Doubre et al., Phys. Rev. C $\underline{15}, 693$ (1977).

${ }^{13}$ W. W. Wilcke et al., Phys. Rev. C $\underline{22}, 128$ (1980). 